\title{
Assisted Reproductive Technology and Retinopathy of Prematurity
}

\author{
Raazia Tanveer ${ }^{1}$, Imrana Abid ${ }^{2}$ \\ ${ }^{1,2}$ Department of Gynecology, Madinat Zayed Hospital, Abu Dhabi. UAE
}

The first pregnancy as a result of in vitro fertilization (IVF) in humans was reported in 1976 and later in 1978, the first IVF baby was born. ${ }^{1}$ Since then, millions of pregnancies have occurred worldwide by IVF and other modifications of this technology. All these procedures are commonly termed assisted reproductive technology or ART. The most common techniques are; intracytoplasmic sperm injection (ICSI), zygote intra-fallopian transfer (ZIFT) and gamete intra-fallopian transfer (GIFT). With more and more experience and advancement in technology, the success rates of ART have increased. Not only that, the indications for these procedures have also changed. Research in this field is complicated by the need to distinguish between the effects of ART on outcomes in offspring versus multiple other confounding or mediating factors. Furthermore, these outcomes are influenced by technological changes in the performance of ART, as well as changes in obstetric and neonatal care, over time.

ART related pregnancies very often result in multiple pregnancies, which are more prone to result in preterm labour. It was reported that in the United States, multiple pregnancies mostly occur as a result of infertility treatments. ${ }^{2}$ The preterm birth rate ranges from 5 percent in parts of Europe to 18 percent in Africa. It was also reported that approximately 15 million pre-term children are born every year. ${ }^{3,4}$ The risk of preterm delivery is higher for multiple pregnancies compared with singletons and it increases

How to Cite this Article: Tanveer R, Abid I. Editorial: Assisted Reproductive Technology and Retinopathy of Prematurity. Pak J Ophthalmol. 2020; 36 (4): 315-317.

Doi: https://doi.org/10.36351/pjo.v36i4.1130

Correspondence: Imrana Abid

Specialist OBGY at Madinat Zayed Hospital

Abu Dhabi. UAE

Email:Imranaabid@yahoo.com with each additional infant. In 2013, the incidence of prematurity in the United States was 57, 93, 96, and 100 percent for twins, triplets, quadruplets, and quintuplets, respectively. ${ }^{5,6}$ Similarly, the incidence of very low birth weight (VLBW) infants (birth weight $<1500 \mathrm{~g}$ ) rose as the number of infants increased; 10 , 37,67 , and 88 percent for twins, triplets, quadruplets, and quintuplets and higher order multiples,

If an infant is born before 37 completed weeks (less than 259 days) of gestation, it is termed as premature birth. Mortality rate of infants born at or before 25 weeks of gestation is quite high. Even if they manage to survive, there is a huge risk of complications including retinopathy of prematurity. ${ }^{6}$

One classification based upon birth weight (BW) includes the following categories:

- Low birth weight (LBW) - BW less than $2500 \mathrm{~g}$.

- Very low birth weight (VLBW) - BW less than $1500 \mathrm{~g}$.

- Extremely low birth weight (ELBW) - BW less than $1000 \mathrm{~g}$.

Prematurity is also defined by Gestational Age (GA) as follows:

- Late preterm infants - GA between 34 weeks and 36 weeks and 6 days.

- Moderate preterm infants - GA between 32 weeks and 33 weeks and 6 days.

- Very preterm (VPT) infants - GA at or below 32 weeks.

- Extremely preterm (EPT) infants - GA at or below 28 weeks.

Preterm infants are at risk for developing short and long-term complications that result from anatomic or functional immaturity during the neonatal period. With decreasing gestational age (GA) and birth weight (BW), chances of complications of prematurity increase which definitely includes Retinopathy of 
prematurity (ROP).

ROP is a retinal pathology, which occurs as a result of premature retina, post-natal hyperoxia and later retinal ischemia. The lesser the gestational age, greater is the incidence and severity of ROP. Although the disease may be experienced as early as 30 to 32 weeks, it typically begins at 34 weeks postmenstrual age (PMA). ROP progresses until 40 to 45 weeks PMA and may subsequently resolve in majority of the infants. ${ }^{7}$ It is less commonly seen in infants with birth weight more than $1500 \mathrm{~g}$ and/or gestational age greater than 32 weeks. However, different geographical areas have varying data regarding its prevalence. The severity of ROP leading to blindness is more in infants of birthweight less than $1000 \mathrm{~g}$.

The environment is relatively hyperoxic to the premature infant after birth, even if there is no supplemental oxygen. The choroidal circulation is not auto-regulated in response to changes, which occur in oxygen saturation after birth. As hyperoxia occurs, more oxygen is diffused from choroid to the retina. Retinal vessels constrict in response to high oxygen. When the eyeball size increases, there is increased demand of oxygen leading to hypoxia and formation of vascular endothelial growth factors. Free radicals, which are formed from oxygen, also play some role in pathogenesis of ROP.

ROP may regress even without treatment. This may occur at any stage of the disease. There can be resultant impairment of vision after regression. If the disease progresses, there are many treatment options which depend upon the stage of the disease at which the diagnosis is made. Earlier the treatment, better are the results. The main idea behind early treatment is to destroy the ischemic retina. At later and advanced stages, retinal surgery may be the answer. ${ }^{8}$ Regressed ROP may present with strabismus, amblyopia, myopia and retarded ocular growth.

In different types of ART, a strong relationship of ROP was seen with IVF. In a meta-analysis, it was found that ART was more commonly associated with stage 3 ROP. ${ }^{9}$ Another report showed that ROP in cases of ART infants was seen even in the heavier newborns. These studies indicated that there may be a need for re-evaluation of the screening protocols. ${ }^{10}$

Although ART has changed the lives of many people, it has increased the burden on neonatologist and ophthalmologists with all types of complications related to prematurity in multiple births. In the last 20 to 25 years, there have been vast improvements in ART and neonatal intensive care, which has resulted in increased survival of most of the immature infants. There are specialized centers, which provide high-risk obstetric care, intensive neonatal care, use of steroids in the prenatal care, improved postnatal treatment with surfactant and nitric oxide and better respirators and advanced equipment for the care of extremely immature infants. All these have contributed to improved survival. Therefore, current changes in neonatal care warrant careful ongoing evaluation during the coming years regarding ROP.

\section{REFERENCES}

1. Steptoe PC, Edwards RG. Birth after the reimplantation of a human embryo. Lancet, 1978; 2: 366.

2. Ory SJ. The national epidemic of multiple pregnancy and the contribution of assisted reproductive technology. Fertil Steril. 2013; 100: 929.

3. WHO, March of Dimes, Partnership for Maternal, Newborn \& Child Health, Save Children. Born too soon: the global action report on preterm birth. Available at:

www.who.int/maternal_child_adolescent/documents/bo rn_too_soon/en/ (Accessed on May 04, 2020).

4. National Vital Statistics Reports. Births: Final data for 2018. 2019; 68 (13). Available at https://www.cdc.gov/nchs/data/nvsr/nvsr68/nvsr68_13_ tables-508.pdf (Accessed on January 16, 2020).

5. Roberts WE, Morrison JC, Hamer C, Wiser WL. The incidence of preterm labor and specific risk factors. Obstet Gynecol. 1990; 76: 85S.

6. Bodeau-Livinec F, Zeitlin J, Blondel B, Arnaud C, Fresson J, A, et al. Do very preterm twins and singletons differ in their neurodevelopment at 5 years of age? Arch Dis Child Fetal Neonatal Ed. 2013; 98 (6): F480-487.

7. Trifonova K, Slaveykov K, Mumdzhiev H, Dzhelebov D. Artificial Reproductive Technology - A Risk Factor for Retinopathy of Prematurity. Open Access Maced J Med Sci. 2018; 6 (11): 2245-2249. Doi: 10.3889/oamjms.2018.448.

8. Connolly BP, Ng EY, McNamara JA, Regillo CD, Vander JF, Tasman W. A comparison of laser photocoagulation with cryotherapy for threshold retinopathy of prematurity at 10 years: Part 2 . Refractive outcome. Ophthalmology, 2002; 109: 936941.

9. Wheatley CM, Dickinson JL, Mackey DA, Craig JE, Sale MM. Retinopathy of prematurity: recent advances in our understanding. Br J Ophthalmol. 2002; 86: 696700 . 
10. Gao L, Shao W, Li Na, Tian C, Jia H, Peng X, et al. The Risk of Retinopathy of Prematurity in the Infants following Assisted Reproductive Technology: A MetaAnalysis. Biomed Res Int. 2019; 2019: 2095730.

Doi: $10.1155 / 2019 / 2095730$.

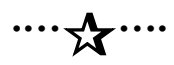

